\title{
Our Interplanetary Bodies
}

Écologie et sublime dans l'œuvre de Tomás Saraceno

Our Interplanetary Bodies: Ecology and Sublime in the work of Tomás

Saraceno

\section{Marie Siguier}

\section{(2) OpenEdition}

\section{Journals}

Édition électronique

URL : http://journals.openedition.org/cel/4139

DOI : $10.4000 /$ cel. 4139

ISSN : 2262-208X

Éditeur

École du Louvre

Référence électronique

Marie Siguier, «Our Interplanetary Bodies », Les Cahiers de l'École du Louvre [En ligne], 14 | 2019, mis en ligne le 02 décembre 2019, consulté le 05 décembre 2019. URL : http://journals.openedition.org/cel/ 4139 ; DOI : 10.4000/cel.4139

Ce document a été généré automatiquement le 5 décembre 2019.

\section{(7) $\odot$}

Les Cahiers de l'École du Louvre sont mis à disposition selon les termes de la licence Creative Commons Attribution - Pas d'Utilisation Commerciale - Pas de Modification 4.0 International. 


\title{
Our Interplanetary Bodies
}

Écologie et sublime dans l'œuvre de Tomás Saraceno

Our Interplanetary Bodies: Ecology and Sublime in the work of Tomás

Saraceno

Marie Siguier

\begin{abstract}
«Les poètes ont créé une lune métaphorique et les savants une lune algébrique. La lune réelle est entre les deux. C'est cette lune que j'avais sous les yeux. [...] Vous vous trouvez face à face dans l'ombre avec cette mappemonde de l'Ignoré [...]. On allait et venait dans cette chimère ; ce centre conjectural d'une création différente de la nôtre était un récipient de vie; on y naissait, on y mourait peut-être ; cette vision était un lien pour lequel nous étions le rêve. Ces hypothèses compliquant une sensation, ces ébauches de la pensée essayée hors du connu, faisaient un chaos dans mon cerveau. [...] L'effet de profondeur et de perte du réel était terrible. Et cependant le réel était là. » Victor Hugo ${ }^{1}$
\end{abstract}

1 Quels modes de connaissances nous permettent-ils de nous introduire à l'intérieur des phénomènes inobservables qui animent l'univers? Comment sommes-nous intégrés en tant qu'êtres humains au sein de la toile cosmique? Existe-t-il des liens invisibles qui nous relient aux multiples entités qui peuplent le monde? Fasciné par ces interrogations à la frontière entre la métaphysique et la science, l'artiste Tomás Saraceno (né en 1973 à San Miguel de Tucumán, Argentine) entend nous sensibiliser dans son exposition Our Interplanetary Bodies à l'ampleur de phénomènes qui outrepassent la perception humaine, lors d'une expérience analogue à celle décrite dans le Promontoire du Songe par Victor Hugo. Mêlant l'art et la biologie à la musique expérimentale, Our Interplanetary Bodies se déploie comme un horizon cosmique où s'hybrident la nature brute et les technologies, à l'Asia Culture Center à Gwangju en Corée du Sud (15 juillet 2017-25 mars 2018). L'artiste pose la question de notre relation au cosmos dans le cadre d'une exposition immersive dont la portée écologique doit se prolonger hors de la sphère muséale et attire notre attention sur l'existence d'entités d'une étendue spatio-temporelle si vaste ou si infime qu'elles déroutent l'idée même que nous nous en faisons.

2 L'espace de près de 1560 mètres carrés a été laissé délibérément à l'état brut par l'artiste - câbles, parois de béton et structures métalliques à nu. De nature 
processuelle, l'exposition varie en permanence dans ses contours les plus imprévisibles, nous invitant à détecter des phénomènes sensoriels habituellement non circonscrits par l'appareil perceptif humain grâce à leur intensification au moyen de dispositifs technologiques. Le régime immersif de présentation des œuvres implique certaines répercussions tant du point de vue de la temporalité, du décentrement spatial et du mouvement au sein d'un environnement tissé par une multiplicité d'acteurs humains et non-humains. Mêlant des présences humaines et animales, des matériaux naturels et technologiques au sein d'un simulacre de cosmos, l'artiste dissout la frontière entre nature, réalité et artificialité.

Our Interplanterary Bodies révèle la persistance de la fascination humaine pour la «nature trop loin », selon l'expression de Victor Hugo et interroge ce qu'il advient de la notion de sublime lorsque notre rapport au monde naturel ne correspond plus au prédicat moderniste. En repartant de l'étymologie allemande du mot das Erhabene (l'élevé), le sublime désigne « ce qui dépasse notre entendement ». Dans la Critique de la faculté de juger ${ }^{2}$ (1790), Kant affirme que le sublime ne désigne ni un objet, ni un spectacle naturel, mais un sentiment éprouvé à l'occasion de certains phénomènes liminaux ou extrêmes de la nature. «L'infinité du cosmos (sublime mathématique de la grandeur) et la puissance naturelle (sublime dynamique de la force) ne sont pas sublimes en elles-mêmes, elles réveillent ou suscitent notre propre sublimité, notre capacité à nous confronter à ce qui nous dépasse absolument ${ }^{3} »$. Selon Andrew Chignell et Matthew C. Halteman, ce sentiment d'élévation a pu être décrit comme: «le (vertige) de rencontrer quelque chose dont les principales caractéristiques dépassent la compréhension intellectuelle, notre esprit étant ainsi élevé au-dessus ou à tout le moins au-delà de ses interactions cognitives typiques avec les objets ${ }^{4}$. " Si au cours des XIX et $\mathrm{xx}^{\mathrm{e}}$ siècles, l'expérience du sublime s'est progressivement déplacée de la nature divine vers la technologie humaine, l'incommensurabilité du cosmos et notre incapacité à saisir les lois qui l'animent assurent la survivance d'une fascination pour le sublime naturel dans notre monde contemporain, tiraillé par les urgences environnementales, climatiques et sociales de l'anthropocène.

4 Cet article est le fruit d'une recherche théorique à la croisée de l'histoire de l'art et de l'esthétique et d'une approche empirique dans le cadre d'une immersion d'un trimestre au sein du studio Tomás Saraceno à Berlin en $2017^{5}$. En partant d'une interprétation phénoménologique de l'exposition, il s'agira d'identifier et d'éclairer les résurgences et dépassements du sublime moderniste au sein de l'œuvre de cet artiste.

En repensant notre rapport au cosmos, Tomás Saraceno participe de la réinvention de la tradition romantique du sublime, mêlée à une conscience aiguë de la transformation de notre univers lié au désastre climatique. Cette démarche confine à une forme de mythologie, renouant avec des récits traditionnels de naissance du monde, faisant sienne l'injonction contemporaine à "replonger dans le "primitif chaos" des sensations pour recréer un monde qui n'est pas le cosmos immuable de nos représentations et formulations habituelles, mais un chaosmose vibrant et vivant ${ }^{6}$.» comme le suggère Michel Collot en lisant Félix Guattari. Plus tôt dans sa carrière, sa série de photographies Cloud Cities, prises sur l'immensité vide du Lac Salar de Uyuni en Bolivie, illustrait la vision d'un artiste en quête de sublime. Cloud Cities met en lumière ce moment de friction au monde dans le point limite de la disparition de l'individu dans le tourment des éléments, dans la plénitude de l'abandon de soi et la contemplation d'un paysage. De même, Evening Sun (2006) thématise le rapport d'osmose entre l'homme et 
la nature entre quête de transcendance et de dissolution $\mathrm{du}$ « je » dans un grand tout cosmique et renvoie l'image d'une humanité diminuée par ses propres inventions et l'expansion sans fin du paysage. Véritable aspiration au voyage céleste, à la perméabilité entre le corps et le monde et à l'a-territorialité héritée des artistes du Land Art traversant les étendues désertiques de l'Ouest américain, ces séries mettent en évidence les rapports étroits qu'entretient Tomás Saraceno avec la tradition romantique et le sublime moderniste. L'expérience du sublime dans Our Interplanetary Bodies est cependant entièrement repensée à l'aune de l'introduction dans notre environnement et dans le régime même de l'œuvre d'art des nouvelles technologies et agentivités qui renouvellent irréversiblement nos interactions avec notre milieu et avec la nature, ainsi que notre compréhension du monde. Qu'advient-il de l'idée de la distance entre le sujet et le monde au principe du sublime moderniste dans une œuvre monumentale conçue comme un environnement inclusif qui mêle la nature à la technologie et qui suppose la rencontre des tous les corps en présence?

\section{Vers un paradigme scientifique de l'art}

6 Les recherches de Tomás Saraceno prennent source dans le mouvement des Subaltern Studies qui conteste largement les effets de domination. Dans le sillage d'Arne Naess, théoricien de «l'écologie profonde » ou deep ecology (1972), il aspire à une nouvelle conception métaphysique où l'humanité entrerait dans un rapport de codépendance et de coexistence avec les non-humains. Sa pratique artistique est tissée de faisceaux de recherches, d'expérimentations, de matériaux et d'échelles qui se métamorphosent et s'agglomèrent en de nouvelles configurations. S'enracinant dans les sciences de la vie et l'astronomie, elle se déploie dans le domaine de l'architecture visionnaire, de l'art et de la musique, bifurque en direction de la science-fiction et de la cosmologie, croise le chemin des sciences sociales, prospectives, de la biologie, de la thermodynamique, mais aussi de la politique et de la philosophie pour atteindre une portée éthique. Opérant par mimétisme au regard de la recherche scientifique, sa pratique est attentive aux sciences du vivant, à l'éthologie, à l'astrophysique qu'il articule à l'architecture et à l'art, ses disciplines d'origine. C'est l'imprégnation de nébuleuses d'idées et de lectures qui constitue la première phase du processus de réflexion de l'artiste et de son atelier de près de quatre-vingts collaborateurs (arachnologues, architectes, philosophes, historiens de l'art, techniciens, artistes, scientifiques). Ce modèle emprunte à l'agence d'architecture et s'avère symptomatique du Collaborative Turn définit par Okwui Enwezor dans la revue Art Forum en 2009. Vient ensuite le temps de la conversation, de l'échange entre l'artiste et les penseurs de son temps ou ceux dont les livres remplissent les étages de sa bibliothèque personnelle; conversations mentales avec Buckminster Fuller, Donna Haraway, Brian Massumi, Jussi Parrika, Deleuze et Guattari ou réelles avec Bruno Latour, Caroline Jones ou Frei Otto... La figure de Tomás Saraceno se dessine comme celle d'un artiste-laborantin au sein de son atelier, véritable machine conceptuelle et foisonnante qui matérialise cette pensée métaphorique. 


\section{Cosmos artificiel et présence de la nature. Un phénomène hybride}

7 Métaphore des astres dans notre système solaire, trois vastes sculptures aériennes émettant une lumière subtile s'élèvent depuis les profondeurs de la galerie et convoquent l'image d'habitats flottants librement dans le cosmos. Celles-ci sont composées de corps sphériques interconnectés, faiblement éclairés de l'intérieur et encastrés dans un réseau de fil qui les maintient ensemble. Simultanément, une grande projection vidéo affiche le mouvement en temps réel de la poussière cosmique contenue dans l'air, illuminée par un rayon de lumière, tandis qu'un système sonore transforme ce mouvement en une expérience audio-spatiale accrue par la composition acoustique à basse fréquence créée par le mouvement d'une araignée Nephila filant sa toile dans un cadre métallique.

En recréant ce paysage crépusculaire, son fond sonore et un microclimat artificiel à l'intérieur du musée, Tomás Saraceno théâtralise la transition de l'extérieur à l'intérieur, de l'ordinaire à l'extraordinaire qui caractérise l'expérience du sublime. En explorant les capacités sensorielles et leurs ancrages culturels, il n'impose pas une lecture normative de l'œuvre et n'évoque pas précisément l'idée de sublime, si tant est qu'il l'ait lui-même consciemment thématisée. Ce paysage cosmique apparaît comme un phénomène construit par l'artiste lui-même qui assume son caractère hybride et artificiel, incluant la présence d'entités vivantes imperceptibles. Cette "nature » est impalpable, car éclatée en une infinité de particules de poussières cosmiques, un nuage de grains de chondrites que l'on ne perçoit pas immédiatement en parcourant l'espace. Alors que le sublime moderniste était illusionniste et tentait de mettre à distance l'observateur d'une nature terrifiante pour lui faire éprouver un sentiment d'effroi et d'élévation morale, le dispositif technologique de Saraceno englobe l'individu et le renvoie à sa propre expérience physique et cognitive de l'environnement, de l'échelle microscopique à macrocosmique. Conçu comme un dispositif cognitif et phénoménologique, Our Interplanetary Bodies renvoie à la représentation d'un univers dont la mécanique artificielle est quant à elle bien visible, bien plus qu'à une vision mimétique du cosmos. Sa lumière irréelle et son microclimat pressurisé sont autant de subterfuges parfaitement identifiables.

\section{Les visions allégoriques du tissu cosmique}

Un faisceau de lumière brille sur un cadre métallique dépourvu de parois de verre dans lequel une araignée tisse sous nos yeux les réseaux de fils. L'observation, dans l'obscurité d'autres espèces agit comme un premier acte de décentrement de l'idiosyncrasie du sujet. Élevée par Saraceno au rang d'un objet artistique en continuelle mutation, elle met en scène l'impression d'assister à la naissance de l'univers. Construite en amont de l'exposition, en partie par des araignées sociales, une petite minorité parmi les insectes solitaires, cette structure unique, multigénérationnelle et multiespèce ne se produirait pas dans la nature. Ces toiles sont comme des structures en miniature permettant de toucher à une compréhension affinée et sensible du réel. Depuis le début des années 2000, Tomás Saraceno mène une recherche sur le comportement et la sociabilité chez différentes espèces d'araignées et une étude approfondie des propriétés structurelles et de traction de leurs toiles, en 
particulier les toiles hybrides, co-constituées par deux ou trois types d'araignées semisociales, sociables ou solitaires, étant lui-même fasciné par leur dynamique d'engendrement dont les logiques résistent à toute explication scientifique.

En s'appuyant sur l'analogie visuelle entre les macrostructures cosmiques et les microstructures arachnéennes, il souhaite cartographier les premiers instants connus du cosmos en spéculant que les araignées perçoivent, connaissent et interagissent avec la matière noire lorsqu'elles forment leur toile. Selon lui, s'il advenait un retournement irréversible de l'anthropocentrisme de la science, nous pourrions regarder les araignées comme une espèce archaïque, mais d'une sensibilité extrême à des formes de la vie qui nous sont encore inaccessibles. Par une déstabilisation ontologique du statut de l'œuvre d'art, ici, une simple construction de la nature, il nous offre un objet pour saisir ce que pourrait être l'image abyssale du passé cosmique que nous contemplons lorsque nous portons notre regard vers les étoiles.

\section{Morphogenèse d'une fable scientifique}

11 Si la démarche artistique de Saraceno met en tension une forme d'esthétisation de la science qu'il articule à une méthodologie de recherche et des expérimentations rigoureuses menées avec des collaborateurs scientifiques, elle repose également sur la construction d'une fable pseudo-scientifique empreinte de sublime et de merveilleux. Son approche spéculative du vivant bifurque en deux voies: celle de l'esthétique du sublime et la rigueur de l'observation scientifique. Dans son œuvre, la recherche scientifique est fertilisée par la quête métaphysique des origines et se nourrit d'un rapport poétique aux modèles scientifiques.

Ainsi, la connexion entre les formes et les savoirs se matérialise-t-elle chez Tomás Saraceno sous la forme de la toile d'araignée qui correspondrait aux $95 \%$ de l'univers qu'il nous reste à découvrir. Le monde sensible de l'araignée est une altérité inconnue, un univers perceptif tissé à travers la toile inexpérimentée par les humains. L'univers en expansion s'étire dans une temporalité cosmique inconcevable. Quelle est la relation entre le cosmos vibratoire de l'araignée Nephila et celle dans laquelle se déplace notre galaxie parmi les filaments et les nébuleuses célestes ? s'interroge l'artiste. Comment rendre compte de ces phénomènes si ce n'est par un travail de transposition d'un monde à un autre?

Cette fiction s'enracine sur les connaissances scientifiques de l'artiste qui recherche par l'imagination le moment où elles s'épuisent. L'art vient combler cet indicible par l'exercice de l'imagination et de la représentation. Or, Jean-François Lyotard dans Qu'est-ce que le postmodernisme? a définit les conditions favorables pour faire éprouver au sujet le sentiment de sublime face à ce qu'il désigne comme l'imprésentable :

«Le sublime [...] a lieu quand, au contraire l'imagination échoue à présenter un objet qui vienne, ne serait-ce qu'en principe, s'accorder avec un concept. Nous avons l'Idée du monde (la totalité de ce qui est), mais nous n'avons pas la capacité d'en montrer un exemple. [...] Nous pouvons concevoir l'absolument grand, l'absolument puissant, mais toute présentation d'un objet destiné à "faire voir" cette grandeur ou cette puissance absolue nous apparaît comme douloureusement insuffisante. Ce sont là des Idées dont il n'y a pas de présentation possible, elles ne font donc rien connaître de la réalité (l'expérience), elles interdisent aussi l'accord libre des facultés qui produit le sentiment du beau, elles empêchent la formation et la stabilisation du goût. On peut les dire imprésentables ${ }^{7} »$. 
Ces phénomènes «imprésentables» du cosmos, Saraceno tente de les représenter en transposant le monde physique et naturel dans la sphère artistique. Se superposant aux entrelacs délicats des architectures arachnéennes, une immense projection vidéo apparait, restituant les trajectoires aléatoires des particules de chondrite. Aussi anciennes que l'univers, « ces poussières cosmiques » seraient des restes d'événements passés et présents. Issues du morcellement des météorites, les particules de chondrite sont aussi âgées que les 4,6 milliards d'années de notre système solaire. Pour Saraceno, le temps de l'araignée - l'arachnochronia - est connecté au temps cosmique, le temps profond des origines. Nous serions nous-mêmes constitués de poussière cosmique. Nous serions faits de la même matière que les galaxies. La toile d'araignée devient un objet esthétique qui paradoxalement a pour visée d'objectiver les équations et modèles des astrophysiciens. Grâce à ces poussières disséminées dans l'air et à ses toiles, Saraceno crée des supports métaphoriques pour simuler l'accrétion de la Terre, qui selon les scientifiques aurait commencé il y a donc près de 4,6 milliards d'années. Cependant, pour Pierre Montebello, la « Terre ne possède pas un âge en soi, mais elle en a un dans un contexte astronomique, géologique et physique que font apparaitre les sciences expérimentales et non les mathématiques ${ }^{8}$." Avec poésie, Saraceno écrit un récit au sein duquel, l'œuvre d'art devient ce monde intemporel où le temps originel, celui des premières traces d'apparitions humaines et le temps futurologique, expression d'un monde scientifique tourné vers la conquête des étoiles et du ciel, se fondent l'un dans l'autre. Or, selon Arnauld Pierre, le mythe moderne d'une maternité cosmique ${ }^{9}$ s'est défini au croisement de l'évolutionnisme et de l'astronomie à travers un matériau scientifique, poétique, littéraire et artistique. Caroline Jones considère que cette appréhension artistique du cosmos par l'artiste repose sur les ressorts de la bio-fiction : «les araignées et la poussière cosmique sont réelles, mais leur narration et leur sonification par Tomás Saraceno créent d'importants contextes fictionnels ${ }^{10}$ ».

\section{Sublime mathématique des milliards de données. Virtualisation de la nature}

Au fil de la déambulation entre les astres, il apparaît plus clairement que le sublime dans Our Interplanetary Bodies se décline sur divers modes d'apparitions. Au-devant de la projection, un autre rayon lumineux révèle des poussières cosmiques perceptibles dans l'espace réel par la médiation de l'éclairage artificiel dirigé sur elles. Ce dispositif intitulé Cosmic Dust tracking (2017) permet de visualiser sur l'écran les trajectoires des particules de chondrite qui se propagent et se déplacent dans l'atmosphère au gré des courants d'air. Face au faisceau, une caméra suit et enregistre en direct les mouvements de chaque particule, traquée et traduite visuellement à l'écran en temps réel sous la forme d'une entité virtuelle. Dès lors, c'est un sublime abstrait, d'ordre mathématique qui se manifeste. Chaque séquence visuelle est traitée par un algorithme qui définit une piste sonore pour chaque grain de poussière en fonction de sa taille et de sa vitesse. Chaque pixel et sonorité sont calculés selon un principe mathématique ayant son référent dans cet environnement hybride. À cette échelle, les grains de poussière imperceptibles évoquent poétiquement l'agglomération colossale de milliards d'étoiles, qui se compactent dynamiquement dans le vide en expansion. La visualisation des données renvoyant à des entités naturelles sur la projection est un appel au pouvoir de la raison à comprendre l'idée même d'infini dont le code serait le 
vecteur. Les données virtuelles de ces particules invisibles à l'œil nu sont esthétisées et rendues abstraites. Elles évoquent la fascination de l'artiste pour les cartographies de l'univers et l'imagerie des réseaux neuronaux ou des séquences d'ADN. Cette esthétisation des données encodées passe par un travail plastique sur les lignes, les séquences et les couleurs orchestrées dans un flux ininterrompu, que le spectateur est invité à décrypter. La visualisation à l'écran s'inspire également d'une simulation scientifique appelée Millenium Simulation décrivant comment la matière s'est dispersée au tout début de l'Univers, formant des filaments de galaxies et de nuages cosmiques. Lancée en 2005 par des scientifiques spécialisés en cosmologie physique au Max Planck Institute for Astrophysics à Garching en Allemagne afin de comparer des observations et des prédictions théoriques, cette simulation informatique a été développée pour étudier l'évolution de la distribution de la matière noire, la formation des galaxies et des trous noirs dans l'Univers ${ }^{11}$. Comme les images produites par les scientifiques, cette iconographie à l'écran remet en question l'idée même de naturalisme. Les programmes informatiques d'imagerie visuelle permettent aux scientifiques de produire en un instant une image virtuelle de la nature. L'esthétisation du langage numérique rappelle le caractère fantasmatique d'un accès immédiat à la nature telle que le défendaient les Modernes. Saraceno rejoue ce passage de la représentation de la nature à sa virtualisation et dépasse les limites assignées à la sphère artistique en faisant entrer le vivant dans son œuvre. Si la nature est virtualisée, elle est également constitutive du dispositif scénographique en tant que composante de l'œuvre.

Dans Our Interplanetary Bodies, la sublimité de la nature présente, bien qu'impalpable et la sublimité mathématique générique des milliards de données, fusionnent. La distance qui nous tenait éloignés d'un cosmos sublime et inatteignable est ici dissoute. L'infiniment petit - la chondrite - et l'infiniment grand - le cosmos - sont placés sur le même dénominateur commun. L'expérience de l'œuvre est celle de la rencontre entre les êtres vivants, de l'immersion dans une écologie conçue comme entremêlement de relations. Tomás Saraceno affirme le pouvoir des données à connecter l'humain au non-humain dans une perspective redoublée par la qualité de présence du vivant dans l'espace muséal.

\section{Le bruit du fond du monde}

Ce flot ininterrompu d'images et de bruits non assignables - ceux des notes attribuées à chaque particule qui varient et s'amplifient lorsqu'elles entrent en collision entre elles ou avec les personnes présentes dans la pièce - accentue l'hyperstimulation sensorielle de l'installation. Tomás Saraceno dit s'être inspiré du récit des astronautes qui furent fascinés par le silence assourdissant de l'espace cosmique. Comme l'écrit Michel Serres, "en myriades, les choses crient. Souvent sourde aux émissions extraterrestres, l'audition est étonnée par ce qui crie sans nom, sans parler aucune langue. La source de ce cri peut être le bourdonnement, le bruit de fond du monde ${ }^{12}$. " Ces bruits parasites, diffusés sur un ensemble de vingt-cinq haut-parleurs, sont la recréation du bruissement du cosmos et renvoient directement aux images générées par ordinateur. L'artiste donne une matérialité sonore à une entité cosmique, pour la faire résonner comme une note perceptible dans les canaux sensoriels de l'ouïe humaine ou animale. En connectant la toile d'araignée à l'étendue de la Voie lactée, il 
fait référence aux conceptions ésotériques et scientifiques sur la Musique des sphères. De Pythagore à l'astronome Johannes Kepler (1571-1630), les théories musicales engendrèrent un modèle cosmique ${ }^{13}$ fondé sur l'imaginaire d'un ordre qui renverrait à la noble simplicité d'un théorème de géométrie, à la prévisibilité des mouvements des corps célestes, reflet de la perfection essentielle de l'Univers. Influencé par ces conceptions musicales, Saraceno propose une nouvelle orchestration, ouvrant un monde multidimensionnel de vibrations où chacun devient un agent. :

«Ces instruments issus de toiles hybrides pourraient être les premiers grands instruments collectifs! Les araignées jouent ensemble une symphonie complexe... avec le son d'une corde qui se répercute sur toutes les autres cordes... si nous sommes capables de nous "régler" en tant qu'espèce, alors la Terre pourrait se répercuter aussi... et les planètes pourraient capturer notre oscillation acoustique ! Bang... Big! ${ }^{14}$ ", s'exclame-t-il.

18 À travers le médium atmosphérique, Our Interplanetary Bodies devient un instrument pour de tels accords cosmiques. L'interaction entre le son de la poussière flottant dans l'espace, des visiteurs errants et le rythme de l'araignée qui déchire sa toile, contribue à créer une composition musicale collective qui fait raisonner les basses fréquences sonores. Le sublime se manifeste alors dans le cadre d'opérations de traduction et de médiation de l'expérience. La coprésence du vivant et de dispositifs biomédias et technologiques qui se substituent à l'idée de nature nous fait éprouver l'interrelation entre l'humain, le non-humain et la matière et reconfigure notre rapport au monde. Saraceno interroge les limites des sens du spectateur, l'incitant à interagir avec des entités naturelles impalpables, sans la médiatisation des technologies. Il substitue la technologie à la nature pour mieux la révéler.

\section{Stratifications spatiotemporelles : espace disjoint et désorientation}

19 Pourtant sobre et unifié par son atmosphère nocturne, l'espace scénographique conçu par l'artiste s'avère intrinsèquement disjoint et fragmenté. Du fait des jeux d'échelles et de découpes successives dans la galerie, la réalité sensible de l'œuvre oscille constamment entre proximité, distance, opacité et transparence. Lorsqu'il se dirige à nouveau vers ce paysage cosmomorphe, le visiteur découvre une multiplicité de perspectives qui renforcent ce sentiment d'immensité. Sur l'une des trois sphères, une échelle est mise à sa disposition. S'il grimpe, un point de vue sur cette immensité nocturne s'ouvre à lui alors qu'il surplombe les visiteurs en contrebas. La déambulation non linéaire entre les sphères vise une perte des repères spatiotemporels. Tomás Saraceno cherche à traduire les propriétés topologiques globales de l'univers dans espace replié (celui d'une galerie de musée) en nous immergeant dans le flux spatiotemporel de la data et de son infinité mathématique, en nous catapultant dans un cosmos artificiel programmé de toute pièce et en nous rendant sensibles et solidaires aux non-humains et entités cosmiques qui respirent avec nous. L'œuvre confine au sublime en ce sens qu'elle parvient à perturber radicalement la perception du mouvement et de l'espace, de telle sorte que nous n'arrivons plus à traiter cognitivement les données perçues. En cessant de percevoir de façon immédiate ou directe, le spectateur désorienté se met à analyser phénoménologiquement son acte 
même de perception. L'artiste nous pousse vers la perception elle-même afin de saisir le dispositif global autant intellectuellement que dans sa dimension phénoménale.

Si nous adhérons à la fable scientifique que nous propose Tomás Saraceno, nous expérimentons l'incommensurabilité d'un cosmos étrangement autosuffisant qui altère notre rapport à l'espace et au temps. La nature n'est plus présentée comme une réalité observable à distance, nous interagissons avec elle dans un espace hybride et dilaté en présence d'autres êtres. Cet espace ouvert révèle le pouvoir des technologies à nous réapproprier les modalités de nos perceptions - en ce sens, elles sont une extension, un amplificateur de nos sens, tel que le fantasmait déjà Marshall McLuhan ${ }^{15}$ dans les années 1960. Cette perception est mise en partage, toujours selon un principe d'intermédialité et d'hybridation, à l'ensemble des spectateurs qui l'éprouve de manière potentiellement collective. À la transcendance sublime d'un sujet singulier, Saraceno substitue l'intersubjectivité, la mise en partage, en retissant le rhizome de nos relations et interactions avec les non-humains et le cosmos. Il nous aide à projeter nos facultés cognitives et notre imagination et simultanément à repousser les limites de nos sens au contact d'une nature étrangère et hybride.

\section{Un réseau construit par le langage sensible plus qu'humain}

21 Our Interplanetary Bodies actualise la notion de sublime et l'harmonise au projet de basculer vers un monde cosmomorphe. La cacophonie cosmique se répercute sur le public et les acteurs non-humains qui ensemble prennent part activement à cet environnement hybride. L'expérience du sublime à l'œuvre ici tend à multiplier les interconnexions entre l'humain et le non-humain que les technologies renouvellent et à infléchir un changement profond de notre rapport au monde en abdiquant le point de vue anthropocentré.

En tant que fondement d'une compréhension rationnelle du monde, la distance constituait chez Burke, puis chez Kant, une condition du sublime conçu comme une expérience de la supériorité de la raison sur le sensible. À l'heure du désastre climatique, l'idée d'une telle séparation et d'une telle distance entre l'être humain et son environnement paraît caduque. Le sublime contemporain résulte désormais de nos interactions avec notre environnement plutôt que de notre confrontation à celui-ci. Or, c'est tout l'enjeu d'Our Interplanetary Bodies: construire des sphères sociales particulières et produire un modèle de sociabilité qui transcende les registres humains, non-humains et matériels. Saraceno repense les processus de subjectivation du sujet par la mise en place de dispositifs pour accentuer et bouleverser l'expérience sensible à l'échelle de la microsociété des participants. L'œuvre devient un réseau social qui encourage et cultive de nouvelles compositions.

Tomás Saraceno propose une reformulation de l'esthétique du sublime proprement contemporaine où bifurquent l'écologie, les biomédias ${ }^{16}$ et les technologies scientifiques. Ses recherches en faveur d'une appréhension unifiée du cosmos participent d'un mouvement de fond transversal en philosophie, en anthropologie et en sociologie où s'observe et se dessine le passage d'un schème anthropomorphe un schème cosmomorphe. Pour Bruno Latour le cosmos désigne tout ce qui est ; il doit être élargi à l'ensemble des entités non-humaines qui participent aux actions humaines. 
Dans Mille Plateaux ${ }^{17}$, Deleuze et Guattari affirment que le tournant " postromantique » de l'art s'opère lorsque ce ne sont plus «les formes, les matières, ni les thèmes » qui importent, mais les «forces, les densités, les intensités » qui sous-tendent le monde visible. Au sujet de l'esthétique deleuzienne, Montebello écrit ces quelques lignes qui font écho à la conception esthétique de Saraceno :

«De quel art ne pourrait-on dire qu'il me fait passer dans un plan plus large, comme une marée, un océan, un ciel, un infini, par affects, par percepts, par sons? Que l'art ne soit au fond que ce mouvement de nous excentrer de l'humain, que le surgissement d'un monde plus large que l'humain qu'il soit toujours un élargissement de l'humain par un infini à capter, ce sera la position esthétique fondamentale de Deleuze. La honte d'être un homme doit s'entendre ainsi : non pas accusation portée contre l'homme, mais plutôt contre l'homme muré en lui-même, narcissique, projectif, ramenant le monde à lui, enfermant la vie en lui, coupée de l'immensité du monde. Quel motif plus puissant l'esthétique pourrait-elle avoir en effet que de connecter l'homme avec l'immensité du monde, que de recomposer l'homme avec le cosmos, afin de l'élargir à mille vies fragiles, invisibles, insoupçonnées, minuscules ou gigantesques, terribles ou sublimes ? [...] Partout l'art nous reconnecte au cosmos et au temps pur, nébuleuse spatiale et nébuleuse du passé, sous des modes infiniment variés, qui n'ont pourtant qu'un but, élargir notre vie par la vaste puissance de vie dans laquelle nous sommes immergés ${ }^{18}$. »

Our Interplanetary Bodies trace un nouveau plan de composition du monde qui articule les êtres les uns aux autres et fait dialoguer les différences physiques, biologique, anthropologique et culturelle. Artiste activiste, fervent défenseur des mondes atmosphériques, Tomás Saraceno nous amène à penser dans une perspective écosophique $^{19}$, c'est-à-dire en termes de complexité, de relation, de rencontre et de coexistence. Sa démarche artistique repose sur la réconciliation de l'esthétique du sublime et de la rigueur de l'observation scientifique et parvient à s'ériger en cosmopolitique : "la cosmopolitique [...] relève d'un sens "spéculatif" fort, elle nous astreint à penser problématiquement le rapport d'obligation et de coexistence entre les êtres et les pratiques qui les convoquent ${ }^{20}$ ». Prenant ses distances vis-à-vis du dualisme moderne qui fonde le sublime naturaliste établi sur un rapport distancié à la nature mystérieuse et terrifiante, Saraceno redistribue les valeurs attribuées au sujet et à l'objet dans l'expérience du sublime. L'expérience de décentrement du sujet et de ses modes d'apprentissage, ainsi que l'observation d'autres espèces nous conduisent à réapprendre à sentir le monde et à calibrer notre présence. En proposant une expérience d'élévation intersubjective, Our Interplanetary Bodies rend poreuse la limite entre la subjectivité et le cosmos et en appel à un nouvel humanisme fondé sur une vision cosmomorphe du monde. 
Fig. 1 à 4
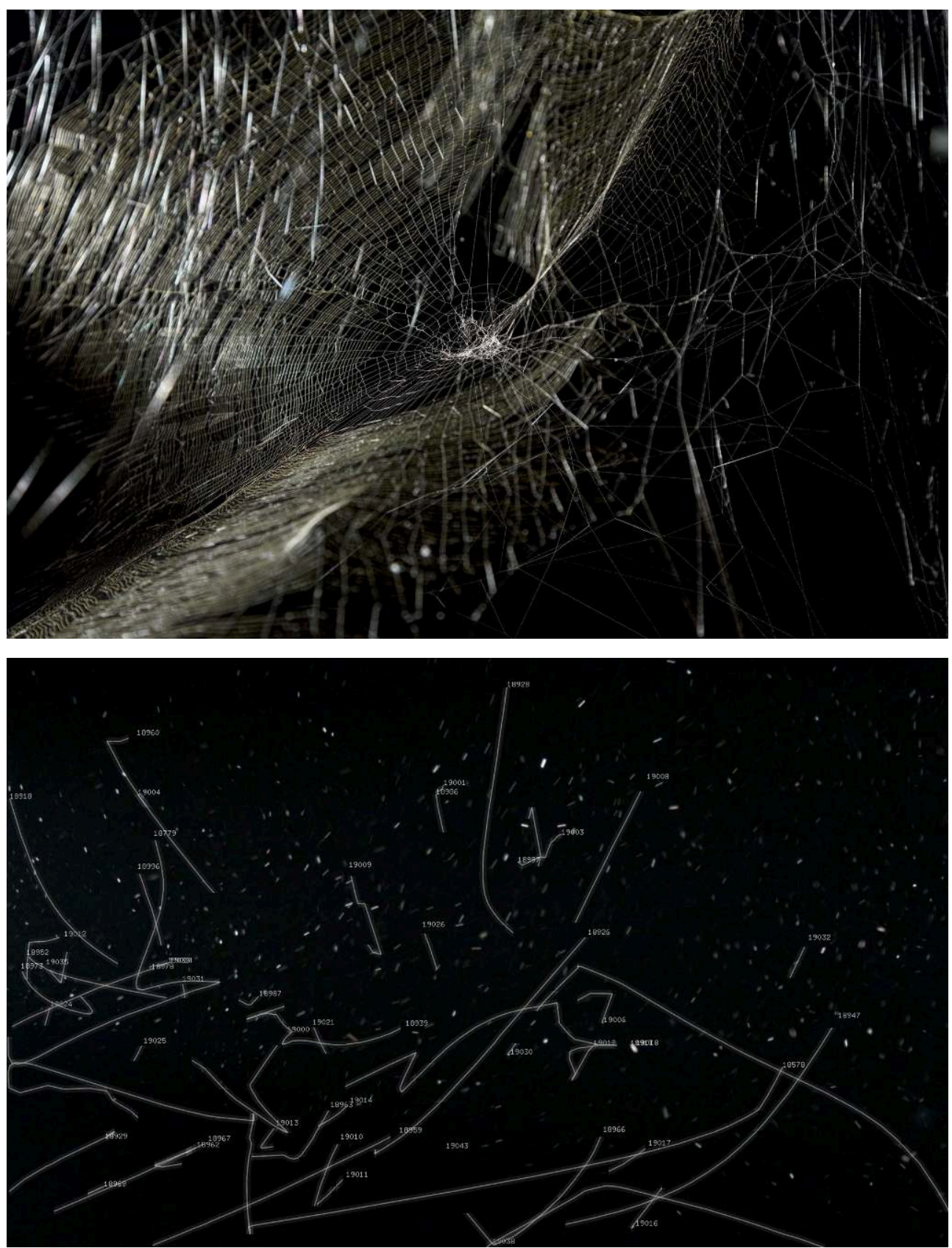

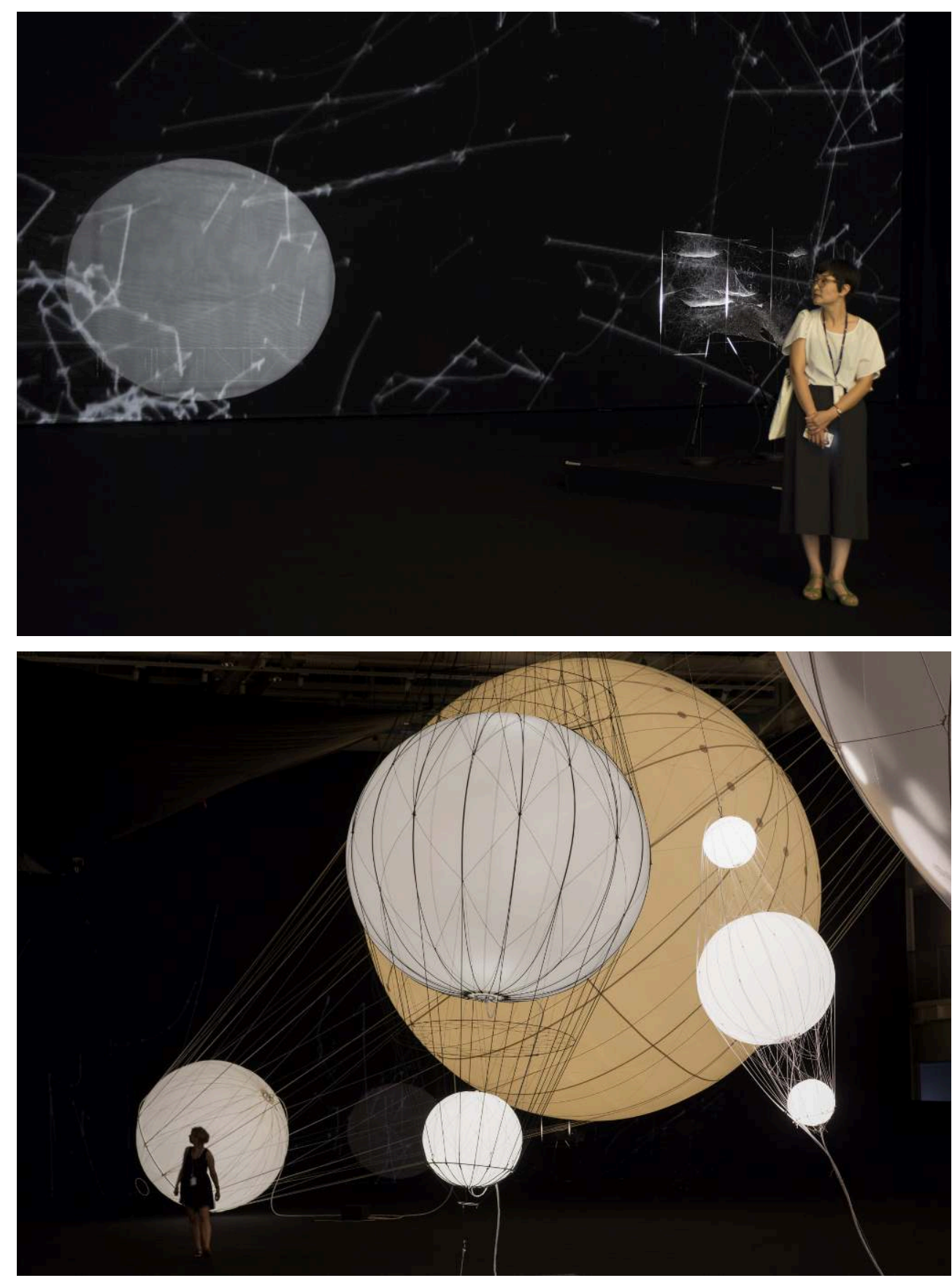

Our Interplanetary Bodies, 2017. Solo exhibition at the Asia Culture Centre, curated by Sung Wong Kim. Courtesy the artist; Tanya Bonakdar Gallery, New York; Andersen's Contemporary, Copenhagen; Pinksummer contemporary art, Genoa; Esther Schipper, Berlin.

(c) Photo Studio Tomas Saraceno, 2017 


\section{NOTES}

1. En 1834, Victor Hugo est invité par l'astronome et physicien François Arago à observer les phases de la Lune. Il décrit cette expérience dans Le Promontoire du songe, Paris, Gallimard, 2012, p. 21.

2. Kant E., La critique de la faculté de juger (1790), Paris, Flammarion, 2000.

3. Sur la question de la relation entre sublime et cosmos du romantisme à l'avantgarde, nous nous référons à l'article d'olivier Scheffer, Cosmos et Sublime in Guénin H. (dir.), Sublime : les tremblements du monde, cat. expo., Centre Pompidou Metz, 11 février 5 septembre 2016, Metz, Centre Pompidou Metz, 2016, p. 26-31.

4. Andrew Chignell et Matthew C. Halteman, "Religion and the Sublime" in Costello (dir.) The Sublime: From Antiquity to the Present, Cambridge University Press, Cambridge, 2012. Andrew Chignell est professeur agrégé à l'Université Cornell. Ses recherches portent sur Kant et d'autres philosophes des débuts de la philosophie moderne, l'épistémologie, l'esthétique et la philosophie de la religion. Matthew C. Halteman est professeur agrégé de philosophie au Calvin College et membre du Oxford Centre for Animal Ethics. Il est l'auteur de Compassionate Eating as Care of Creation (2008).

5. En 2017, j'ai collaboré en tant qu'assistante éditoriale effectuant en parallèle mes recherches sur la pratique artistique de cet artiste dans le cadre de mon mémoire Vibrer avec le cosmos. Les trajectoires écosophiques du Studio Tomás Saraceno. C'est lors de cette expérience que j'ai participé à l'élaboration de l'exposition Our Interplanetary Bodies.

6. Collot M. «Poésie, Paysage et sensation », [En ligne], consulté le 13.06.19: http:// oaji.net/articles/2017/6266-1538046429.pdf Né en 1952, Michel Collot est un poète, critique littéraire français, spécialiste de la poésie française moderne et contemporaine et des représentations artistiques et littéraires du paysage. Il est professeur émérite en littéraire française à l'Université Sorbonne-Nouvelle Paris 3.

7. Lyotard J-F., « Qu'est-ce que le postmodernisme ? ", Critique n419, Paris, 1982.

8. Montebello P., Métaphysiques cosmomorphes, Dijon, Les Presses du réel, p. 52.

9. Pierre A., Maternités cosmiques. La recherche des origines, de Kupka à Kubrick, Paris, Hazan, 2010.

10. Jones Caroline, "Webbing Umwelten into Chronotopes: Tomás Saraceno, Spiders, and Cosmic Dust" in How to Entangle the Universe in a Spider Web, cat. expo., Buenos Aires, Museo de Arte Moderno de Buenos Aires, 2018.

11. Le groupe Consortium Virgo a procédé en simplifiant les représentations des processus astrophysiques observés pour affecter les galaxies réelles et obtenir une série de simulations sophistiquées de la formation de la population des galaxies, accessibles au public sur Internet.

12. Michel Serres cité in McCormack D., "Sounding: Echoes and Threshold of Atmospheric media", [En ligne], URL : https://pure.royalholloway.ac.uk/portal/files/ 28346560/AeroceneCampusReader_FINALVERSION.pdf (consulté le 16 février 2018).

13. Sur cette question, voir James J., La Musique des sphères, Monaco, Éditions du Rocher, 1997. 
14. Tomás Saraceno dans un email daté du 29 avril 2014, exprimant ses idées pour l'exposition Cosmic Jive: Tomás Saraceno. The Spider Sessions. Pezzato G. (dir.), Cosmic Jive The Spider Sessions, Genève, Asinello Press, 2015.

15. McLuhan, Pour comprendre les médias: Les prolongements technologiques de l'homme, Paris, Seuil, 2013, p. 25.

16. Thacker E., Biomedia, Minneapolis, University of Minnessota Press, 2004. Pour Eugene Thacker, les biomédias résultent de la fusion progressive de l'informatique, de la biologie moléculaire et des codes génétiques et informatiques. Les corps biologiques seraient des instances médiatrices, tout comme les médias peuvent devenir biologicoorganiques. Il souhaite dépasser la division ontologique entre "bio " et "média ", renouvelant ainsi notre conception classique de la technologie, pour la faire fusionner avec le corps. Les biomédias supposent une relation plus étroite entre l'humain, la machine, la biologie et la technologie, la génétique et l'informatique, l'ADN et le code binaire.

17. Deleuze G., Guattari F. (1980), Mille plateaux: capitalisme et Schizophrénie, Paris, Éditions de Minuit, 2009.

18. Montebello P., Deleuze, Esthétiques, La Honte d'être un homme, Dijon, Les Presses du Réel, p. 167.

19. Guattari F., Les Trois écologies, Paris, Galilée, 1989.

20. Montebello P., Métaphysiques cosmomorphes, op.cit., p. 193. Sur cette notion, se référer à Stengers I., Cosmopolitques II, Paris, La Découverte, 2003.

\section{RÉSUMÉS}

L'exposition Our Interplanetary Bodies (2017) de Tomás Saraceno questionne notre relation au cosmos dans une perspective phénoménologique et cosmopolitique. Vaste recréation d'un paysage cosmique, l'œuvre apparaît comme un phénomène hybride et artificiel par la fusion des éléments organiques (poussières, grains de météorites, arachnides, soie d'araignée) et des nouvelles technologies (écrans, dispositifs bioacoustiques, algorithmes). Alors que le sublime moderniste burkien était illusionniste et tentait de mettre à distance l'observateur d'une nature terrifiante pour lui faire éprouver un sentiment d'effroi, d'élévation ou de ravissement, l'installation englobe l'individu et le renvoie à sa propre expérience physique et cognitive, ainsi qu'à une expérience renouvelée de l'espace et des sens par l'immersion dans un "cosmos programmé » qui produit néanmoins un sentiment d'élévation face à ce que Lyotard appelait « l'imprésentable » : l'absolument grand, l'absolument puissant.

Tomás Saraceno's exhibition Our Interplanetary Bodies (2017) examines our relationship to the cosmos from a phenomenological and cosmopolitanist perspective. The vast recreation of a cosmic landscape, the work appears like a hybrid and artificial phenomenon combining organic elements (dust, meteorite seeds, arachnids, spider webs) and new technologies (screens, bioacoustic devices, algorithms). While the sublime Burkean modernist was an illusionist and attempted to distance the observer from a terrifying nature to have him feel terror, elevation or rapture, the installation encompasses the individual and sends him back to his own physical and 
cognitive experience, as well as to an renewed experience of space and senses through immersion in a "cosmos programme" that nevertheless produces a feeling of elevation faced with what Lyotard called "the unpresentable": the absolutely great, the absolutely powerful.

\section{INDEX}

Mots-clés : Sublime, cosmos, écosophie, phénoménologie, vivant, art/science, utopie, bioacoustique, non-humain

Keywords : sublime, cosmos, ecosophy, phenomenology, vivant, art/science, utopia, bioacoustics, non-human

\section{AUTEUR}

\section{MARIE SIGUIER}

Diplômée de l'École du Louvre en esthétique à Sorbonne Université - Faculté de Lettres, Marie Siguier est assistante de projets curatoriaux et éditoriaux auprès du commissaire d'exposition Jérôme Sans. Après plusieurs mois en tant qu'assistante éditoriale au Studio Tomás Saraceno à Berlin, elle soutient son mémoire Vibrer avec le cosmos. Les trajectoires écosophiques du Studio Tomás Saraceno sous la direction de Marie-Ange Brayer, conservatrice en chef du service Design \& Prospective industrielle au MNAM-Cci - Centre Pompidou, qu'elle a assisté sur plusieurs expositions. Elle publie dans des revues et catalogues d'exposition et poursuit ses recherches au croisement de l'art contemporain, de l'architecture et de l'épistémologie des sciences A graduate of the École du Louvre and the Sorbonne Université-Faculté de Lettres (aesthetics), Marie Siguier assists the exhibition curator Jérôme Sans on curatorial and editorial projects. After several months as an editorial assistant at Studio Tomás Saraceno in Berlin, she defended her dissertation "Vibrer avec le cosmos. Les trajectoires écosophiques du Studio Tomás Saraceno", which was supervised by Marie-Ange Brayer, head curator of the Design et Prospective Industrielle department at the MNAM-Cci-Centre Pompidou, whom she assisted on several exhibitions. She contributes to magazines and exhibition catalogues and pursues her research at the crossroads of contemporary art, architecture and the epistemology of science. 